\title{
Possible Involvement of the Transient Receptor Potential Vanilloid Type 1 Channel in Postoperative Adhesive Obstruction and Its Prevention by a Kampo (Traditional Japanese) Medicine, Daikenchuto
}

\author{
Yohei Tokita ${ }^{1, *}$, Masahiro Yamamoto ${ }^{1}$, Kazuko Satoh $^{1}$, Mitsue Nishiyama ${ }^{1}$, Seiichi Iizuka ${ }^{1}$, \\ Sachiko Imamura ${ }^{1}$, and Yoshio Kase ${ }^{1}$ \\ ${ }^{1}$ Tsumura Research Laboratories, Tsumura \& Co., 3586 Yoshiwara, Ami-machi, Inashiki-gun, Ibaraki 300-1192, Japan
}

Received September 2, 2010; Accepted November 26, 2010

\begin{abstract}
This study focused on the localization of transient receptor potential vanilloid type 1 (TRPV1) in the intestines in postoperative adhesion model rats and investigated the underlying mechanism for the anti-adhesion action of daikenchuto (DKT), especially in relation to TRPV1. Postoperative intestinal adhesion was induced by sprinkling talc in the small intestine. The expression of TRPV1 mRNA was examined by in situ hybridization and real-time RT-PCR. The effects of DKT and its major ingredient, hydroxy sanshool, with or without ruthenium red, a TRP-channel antagonist, on talc-induced intestinal adhesions were evaluated. The level of TRPV1 mRNA was higher in the adhesion regions of talc-treated rats than in normal small intestine of sham-operated rats. Localization of TRPV1 mRNA expression was identified in the submucosal plexus of both sham-operated and talc-treated rats; and in talc-treated rats, it was observed also in the myenteric plexus and regions of adhesion. Capsaicin, DKT, and hydroxy sanshool significantly prevented formation of intestinal adhesions. The effects of DKT and hydroxy sanshool were abrogated by subcutaneous injection of ruthenium red. These results suggest that pharmacological modulation of TRPV1 might be a possible therapeutic option in postoperative intestinal adhesion, which might be relevant to the prevention of postoperative adhesive obstruction by DKT.
\end{abstract}

Keywords: transient receptor potential vanilloid type 1 (TRPV1), sensory nerve, postoperative adhesion, daikenchuto, hydroxy sanshool

\section{Introduction}

Postoperative adhesive obstruction is a cause of postoperative morbidity and remains one of the major longterm complications associated with surgery and lengthy hospital stays $(1,2)$. In particular, postoperative adhesion frequently leads to chronic abdominal pain (3). It is thought to be caused by restricting organ mobility or expansibility and thus stimulating stretch receptors in the smooth muscle of adjacent organs or the abdominal wall $(4,5)$. Our previous report indicated that postoperative intestinal adhesion is complicated by changes in the cholinergic nervous system and that alterations of gastro-

*Corresponding author. tokita youhei@mail.tsumura.co.jp Published online in J-STAGE on December 21, 2010 (in advance) doi: 10.1254/jphs. 10226FP intestinal function might cause adhesion formation (6). Moreover, sensory nerves have been identified histologically in human peritoneal adhesion tissue, suggesting that sensory nerves may be involved in modulation of neurogenic inflammation and chronic pain (7). However, sensory nerves in the adhesion region are essential to the healing of postoperative wounds (8), and further studies are necessary to clarify the role of the sensory nerves in postoperative intestinal adhesion.

In the gastrointestinal tract, transient receptor potential vanilloid type 1 (TRPV1), which was previously recognized as the capsaicin receptor, is expressed primarily in the regions associated with sensory neurons such as axons of spinal afferent neurons and to some extent, vagal afferents $(9,10)$. TRPV1 plays important roles not only in hypersensitivity to inflammatory pain but also in protection of the mucosa in the gastrointestinal tract $(10$, 
11). The stimulation of sensory nerves by capsaicin has been reported to attenuate gastrointestinal damage due to various injurious factors (12). Furthermore, stimulation of TRPV1 promotes neurogenic inflammation in the peripheral tissue via neuropeptides from sensory neurons (13). In particular, inflammatory bowel disease is associated with up-regulation of TRPV1 in nerve fibers of the colon (14). There is a possibility that the inflammation induces a change in the expression and alters the function of TRPV1 on the sensory nerves.

The kampo (traditional Japanese) medicine daikenchuto (DKT) has been integrated into the modern Japanese medical system as a pharmaceutical grade ethical drug and is widely prescribed for patients with dysfunctional gut disorders such as postoperative ileus and gut-adhesive obstruction following abdominal surgery (15). In a double-blind, randomized, placebo-controlled study of healthy humans whose gastrointestinal transit and colonic transit were measured by scintigraphy, DKT had a pro-motility effect, especially acceleration of the ascending colon emptying half-time (16). In experimental studies, DKT showed a preventive effect in talc-induced postoperative intestinal adhesion $(17,18)$ and colonic adhesion due to trinitrobenzenesulfonic acid-induced colitis (19). DKT is composed of four medical herbs including zanthoxylum fruit, processed ginger, ginseng, and maltose syrup. Among these component herbs, processed ginger and zanthoxylum fruit are known to contain various agonistic ligands for TRP channels such as gingerols and hydroxy sanshools $(20,21)$.

Taken together, these findings suggest that neurogenic inflammation via TRP-channel pathways may be involved in postoperative adhesive obstruction. This study focused on TRPV1, which is known to be present predominantly in sensory nerves and investigated the preventive effect of capsaicin and DKT on postoperative intestinal adhesion, especially in relation to TRP channels.

\section{Materials and Methods}

\section{Animals}

Six-week-old male Sprague-Dawley rats were purchased from Charles River Laboratories Japan, Inc. (Yokohama). The animals were allowed free access to water and standard laboratory food (MF; Oriental Yeast, Tokyo) and housed in an animal room kept at a temperature of $23 \pm 1^{\circ} \mathrm{C}$, a relative humidity of $55 \pm 5 \%$, and controlled lighting with the lights on from 7:00 to 19:00 daily.

All experimental procedures were performed according to the 'Guidelines for the Care and Use of Laboratory Animals' approved by the Laboratory Animal Commit- tee of Tsumura \& Co.

\section{Reagents}

Talc and capsaicin were purchased from Wako Pure Chemicals Industries (Osaka), and ruthenium red (RR) was purchased from Sigma-Aldrich Chemical Co. (St. Louis, MO, USA). Hydroxy sanshool was obtained from Tsumura \& Co. (Tokyo). Other reagents used for analysis were purchased from commercial sources.

DKT extract (Lot No. 2009999100, 2040100010), processed ginger (Zingiber officinale Roscoe, rhizome, Lot No. 2041067010), ginseng (Araliaceae, Panax ginseng C.A. Meyer, radix, Lot No. 2041015010), and zanthoxylum fruit (Rutaceae, Zanthoxylum piperitum $\mathrm{DE}$ Candolle, Lot No. 2041094010) were obtained from Tsumura \& Co. in the form of a dried powder. DKT extract powder is manufactured as an aqueous extract containing processed ginger, ginseng, and zanthoxylum fruit in the ratio of 5:3:2. The qualities of these raw materials were tested according to the Japanese Pharmacopoeia and our company's standards. The three medical herbs were extracted with purified water at $95^{\circ} \mathrm{C}$ for $1 \mathrm{~h}$. The extract solution was separated from the non-soluble waste and concentrated by removing water under reduced pressure. Spray-drying was used to produce a dried extract powder. The yield of the extract was $12.5 \%$. DKT is prepared by mixing DKT extract powder and maltose syrup powder (Tsumura \& Co.) at a ratio of 1:8.

\section{Talc-induced intestinal adhesion}

The postoperative intestinal adhesion model was conducted according to a previous report (6). In brief, the rats were fasted overnight but given free access to water before surgery. The rats were anesthetized with ether, and laparotomy was performed using a $3-\mathrm{cm}$ midline incision. The small intestine was gently pulled out of the abdominal cavity and spread. The small intestine was sprinkled uniformly with $50 \mathrm{mg}$ talc and furled like a fan. Then, the small intestine was replaced in the abdominal cavity, and the surgical wound was closed using twolayer continuous sutures. Sham-operated rats were anesthetized with ether, and a $3-\mathrm{cm}$ midline incision was made. The small intestine was gently manipulated, and the surgical wound was closed by two-layer continuous sutures.

\section{Histology}

The histology of talc-induced intestinal adhesion and sham-operated rats was evaluated 7 days after surgery. The small intestine was fixed in $15 \%$ buffered-formalin and examined under a light microscope after embedding in paraffin, sectioning, and staining with hematoxylineosin (HE). 


\section{In situ hybridization (ISH)}

ISH was performed by using the manual capillary action technique with the MicroProbe Staining System; mRNA in sections was hybridized with a specific oligonucleotide antisense probe (Falma, Tokyo). The oligonucleotide probe sequence was used (3'-CTTCAGGCT GTCTGTCTTCCGGGCAACGTC-5'), which is complimentary to the rat vanilloid receptor type 1 mRNA at nucleotides 509 - 539 (22). The sections were deparaffinized and digested with $0.25 \mathrm{mg} / \mathrm{mL}$ pepsin solution diluted with $0.1 \mathrm{~N}$ hydrochloric acid. The sections were hybridized for $30 \mathrm{~min}$ at $45^{\circ} \mathrm{C}$, and post-hybridization washing was performed using standard sodium citrate. The biotin-labeled hybrids were sequentially detected with alkaline phosphatase-conjugated streptavidin for 10 min at $50^{\circ} \mathrm{C}$.

\section{Preparation of total RNA for analysis of TRPV1 $m R N A$ expression}

The experimental animals were killed by decapitation, and the small intestine was removed and placed in icecold saline. The mucus and extraneous tissue were removed on a cold surface. The tissue segments included the longitudinal and circular smooth muscle layers.

Total RNA was extracted from small intestinal tissue using an RNeasy ${ }^{\circledR}$ Mini Kit (Qiagen, Valencia, CA, USA) according to the manufacturer's protocol. Approximately $50 \mathrm{mg}$ of each tissue sample was homogenized in QIAzol lysis reagent (Qiagen $\mathrm{GmbH}$, Hilden, Germany) with two 3-mm diameter beads by using a Micro Smash MS-100-R (Tomy Seiko Co., Ltd., Tokyo) at 3,000 rpm for $3 \mathrm{~min}$. After addition of chloroform, the homogenate was separated into aqueous and organic phases by centrifugation $\left(12,000 \times g, 15 \mathrm{~min}, 4^{\circ} \mathrm{C}\right)$. The upper, aqueous phase was extracted, and ethanol was added to provide appropriate binding conditions. The sample was then applied to the RNeasy Mini Spin Column, in which the total RNA binds to the membrane and phenol and other contaminants are efficiently washed away. Total RNA was eluted in $50 \mu \mathrm{L}$ of RNase-free water. The quantity and purity of the RNA were determined by measuring absorbance at 260 and $280 \mathrm{~nm}$ using a NanoDrop ND-1000 spectrometer (Scrum, Inc., Tokyo).

First-strand $C D N A$ synthesis and quantitative RT-PCR analysis of TRPV1 $\mathrm{mRNA}$ expression

A 1- $\mu \mathrm{g}$ sample of total RNA was reverse-transcribed using a High Capacity cDNA Reverse Transcription Kit (Applied Biosystems, Foster City, CA, USA) following the manufacturer's protocol. The RT products were analyzed by real-time PCR by using TaqMan Gene Expression Master Mix. Real-time PCR was monitored using an
ABI Prism 7900HT (Applied Biosystems) with a TaqMan gene expression assay probe for Trpv1 (probe ID: Rn00583117_m1, Applied Biosystems). Reaction conditions were $50^{\circ} \mathrm{C}$ for $2 \mathrm{~min}$ and then $95^{\circ} \mathrm{C}$ for $10 \mathrm{~min}$ followed by 40 cycles of $95^{\circ} \mathrm{C}$ for $15 \mathrm{~s}$ and $60^{\circ} \mathrm{C}$ for 1 min. Each sample was analyzed in triplicate. $\beta$-Actin (probe ID: Rn00667869_m1) was analyzed as an internal control. An equal volume of a standard was used for each sample. The standard sample was used to make a standard curve for $\beta$-actin, and relative quantifications of the Trpv1 and $\beta$-actin in each sample were determined by using the standard curve. For comparison, Trpv1 values of each sample were normalized to their $\beta$-actin values.

\section{Adhesion}

Adhesion formation was evaluated 7 days after surgery. All rats were sacrificed by decapitation, and the small intestine was removed for analysis. All adhesions were gently separated, and the small intestine was extended to its total length and measured. The adhesion rate was obtained by dividing the length of all adhesions by the total length of the small intestine [(total adhesion length / whole intestinal length) $\times 100]$.

\section{Sensory denervation}

Sensory denervation was performed by the previously reported method (23). Capsaicin was dissolved in a vehicle consisting of $10 \%$ ethanol, $10 \%$ Tween 80 , and $80 \%$ saline. Rats were injected subcutaneously with a total dose of $125 \mathrm{mg} / \mathrm{kg}$ capsaicin over 2 days, with 25 $\mathrm{mg} / \mathrm{kg}$ in the morning and $50 \mathrm{mg} / \mathrm{kg}$ in the afternoon on the first day and $50 \mathrm{mg} / \mathrm{kg}$ on the second day.

Capsaicin injections were performed under ether anesthesia, and the rats were pretreated with terbutaline $(0.1$ $\mathrm{mg} / \mathrm{kg}$, i.m.) and aminophylline $(10 \mathrm{mg} / \mathrm{kg}$, i.m.) to counteract the respiratory impairment associated with capsaicin injection. The rats were used for the experiments at 10 days after treatment. To check the effectiveness of the treatment, a drop of a $0.1 \mathrm{mg} / \mathrm{mL}$ capsaicin solution in saline was instilled into the eye and the reactive wiping movements of the eye were observed. Capsaicin-treated animals that showed any wiping movement were excluded from this study.

\section{Evaluation of drugs}

The rats were allowed to recover $2-3 \mathrm{~h}$ after surgery. Capsaicin, DKT, or one of its components was administered orally once a day for 1 week. On the day following the final administration, the rats were killed by decapitation. The small intestine was removed for analysis, and the adhesion rate was obtained. Moreover, to determine the influence of a TRPV1 antagonist on the effect of capsaicin, RR (1 mg/kg per day) was injected subcutane- 
ously into some rats before test drug administration.

\section{Statistical analyses}

The statistical significance of differences between two groups was assessed by Student's $t$-test. For multiple comparisons between groups, Dunnett's or Fisher's protected least significantly difference (PLSD) test was used. A probability value of less than 0.05 was regarded as significant.

\section{Results}

\section{Histology}

The histology of HE-stained adhesion regions is shown in Fig. 1. No damage was observed histologically in the sham-operated rats. In contrast, infiltration of inflammatory cells, accumulation of fibrin, and necrosis were observed in the adhesion regions on the talc-induced intestinal adhesion rats.

\section{TRPV1 mRNA expression}

To investigate the localization of TRPV1, we detected TRPV1 mRNA by using an in situ hybridization method and real-time RT-PCR in intestinal adhesion regions in the talc-induced intestinal adhesion rats. Expression of TRPV1 mRNA was identified in the submucosal plexus within the muscle layers in sham-operated and talcinduced intestinal adhesion rats. TRPV1 mRNA expres- sion was also identified in the myenteric plexus and inflammatory adhesion regions in talc-induced intestinal adhesion rats, but not in sham-operated rats (Fig. 1). The level of TRPV1 mRNA expression was approximately $44 \%$ higher in adhesion regions in talc-induced intestinal adhesion rats than in the small intestine in sham-operated rats (Fig. 2).

\section{Effect of capsaicin}

The adhesion rate of the control group was markedly

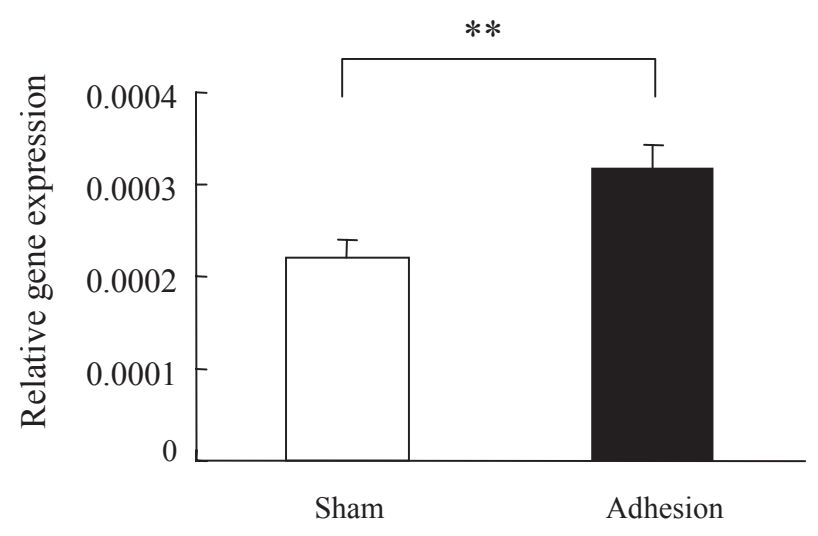

Fig. 2. Expression of TRPV1 mRNA in small intestine (including adhesion regions) isolated from adhesion and sham-operated rats. Each value is the mean \pm S.E.M. of $7-8$ rats. $* * P<0.01$, compared with sham-operated rats (Student's $t$-test).
(A) Sham

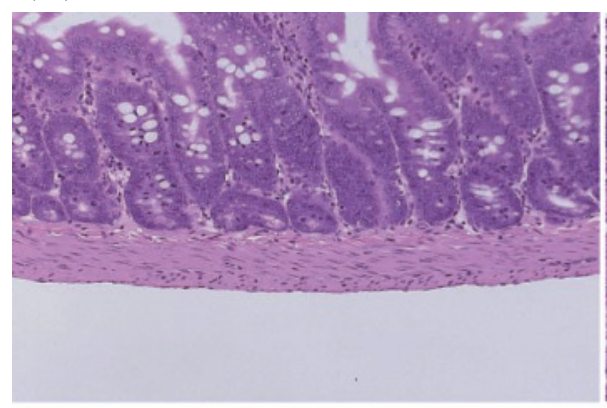

(C) Sham

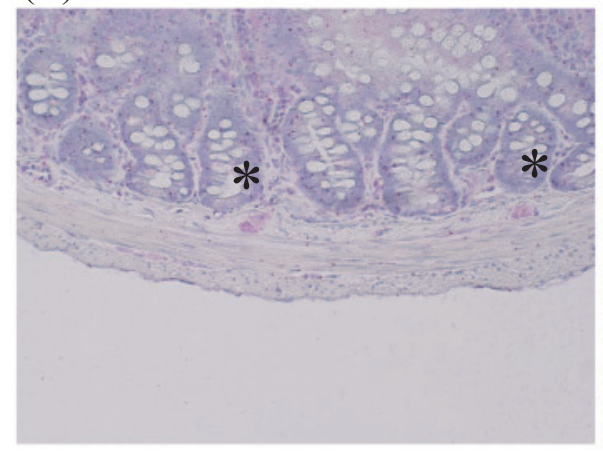

(B) Adhesion

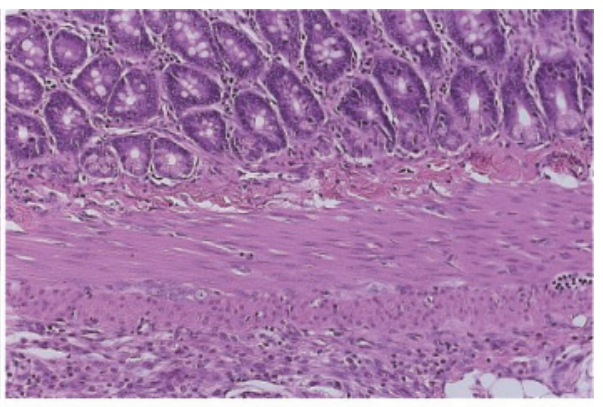

(D) Adhesion

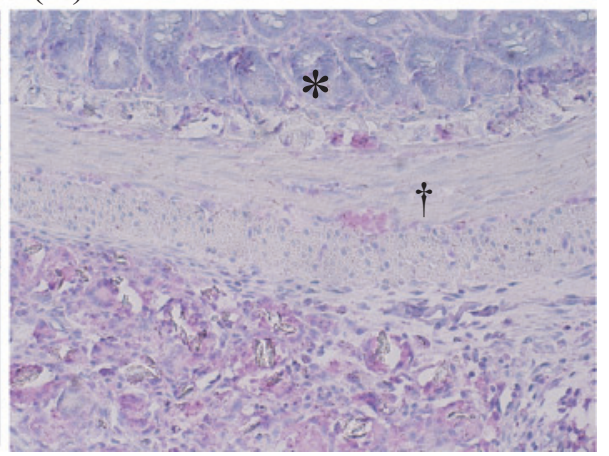

Fig. 1. Histology of talc-induced intestinal adhesion rats (HE stain; original magnification, $\times 50$ ). Sham-operated $(A)$ and talc-induced intestinal adhesion (B) at 7 days after surgery. TRPV1 mRNA expression in the small intestine at 7 days after surgery determined by in situ hybridization histochemistry (original magnification, $\times 50)$. Sham-operated (C) and talcinduced intestinal adhesion (D) at 7 days after surgery. * indicates TRPV1 mRNA expression on the submucosal plexus region. ${ }^{\dagger}$ indicates TRPV1 mRNA expression on the myenteric plexus region. 
increased to $33.8 \pm 4.1 \%$, while adhesion formation was not observed in the sham-operated group. Orally administered capsaicin prevented adhesion formation compared with the control group, and a significant effect was observed at 1 and $3 \mathrm{mg} / \mathrm{kg}$, the adhesion rates being $21.7 \pm 3.2 \%$ and $22.2 \pm 3.2 \%$, respectively (Fig. 3 ).

\section{Effect of TRP-channel antagonist and sensory denerva- tion}

The adhesion rate at a dose of $3 \mathrm{mg} / \mathrm{kg}$ capsaicin was $24.0 \pm 2.1 \%$. Thus, it significantly prevented adhesion formation compared with the control group (the value being $36.9 \pm 3.1 \%$ ), and this effect of capsaicin was completely inhibited by subcutaneous injection of RR. RR did not affect adhesion formation in talc-induced intestinal adhesion rats (Fig. 4A). The adhesion rate in the denervation group (functional ablation of sensory nerves by a large dose of capsaicin) was $19.9 \pm 3.8 \%$, which denotes a significant reduction in adhesion formation compared with the control group (the value being $30.7 \pm 3.8 \%$ ). Capsaicin treatment apparently did not affect the intestinal adhesion in the denervated rats (Fig. 4B).

(A) Sham

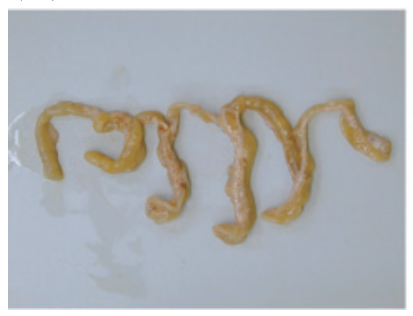

(B) Capsaicin $(3 \mathrm{mg} / \mathrm{kg})$

(C)

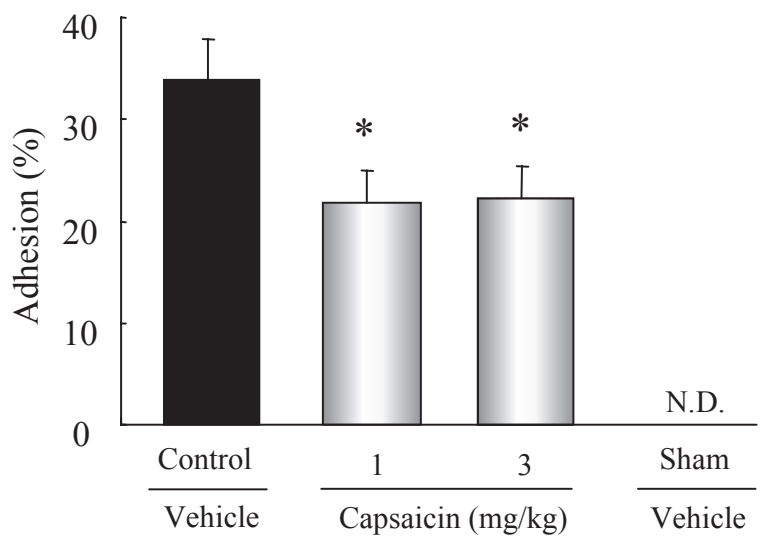

Fig. 3. Gross appearance of talc-induced intestinal adhesion in rats. Control (A) and capsaicin (3 mg/kg)-treated rats (B) at 7 days after surgery. C) Effects of capsaicin (1 and $3 \mathrm{mg} / \mathrm{kg}$ ) on the adhesion rate on the talc-induced intestinal adhesion in rats. Data are expressed as the mean \pm S.E.M. of 13 rats. *Significantly different from the control group at $P<0.05$ (Dunnett's test). N.D., not detected.

\section{Effects of DKT and its components}

At a dose of $2,700 \mathrm{mg} / \mathrm{kg}$, the adhesion rate was $23.6 \pm 3.0 \%$, indicating that DKT significantly prevented adhesion formation compared with the control group (the value being $39.9 \pm 2.9 \%$ ). This effect of DKT was completely inhibited by subcutaneous injection of RR (Fig. 5: C, D). The dose of the constituents of DKT was determined based on the composition of DKT $(2,700 \mathrm{mg} / \mathrm{kg})$. Thus, $60 \mathrm{mg} / \mathrm{kg}$ zanthoxylum fruit, $150 \mathrm{mg} / \mathrm{kg}$ processed ginger, $90 \mathrm{mg} / \mathrm{kg}$ ginseng, and $2,400 \mathrm{mg} / \mathrm{kg}$ maltose syrup were used. The adhesion rates after administration of zanthoxylum fruit, maltose syrup, processed ginger, and ginseng were $26.3 \pm 3.9 \%, 28.2 \pm 3.3 \%, 31.8 \pm 5.4 \%$, and $36.4 \pm 6.4 \%$, respectively (Fig. 6A), the former two having significantly prevented adhesion formation in talc-induced intestinal adhesion rats (the adhesion rate of the control group was $46.0 \pm 5.1 \%$ ). In addition, admin-

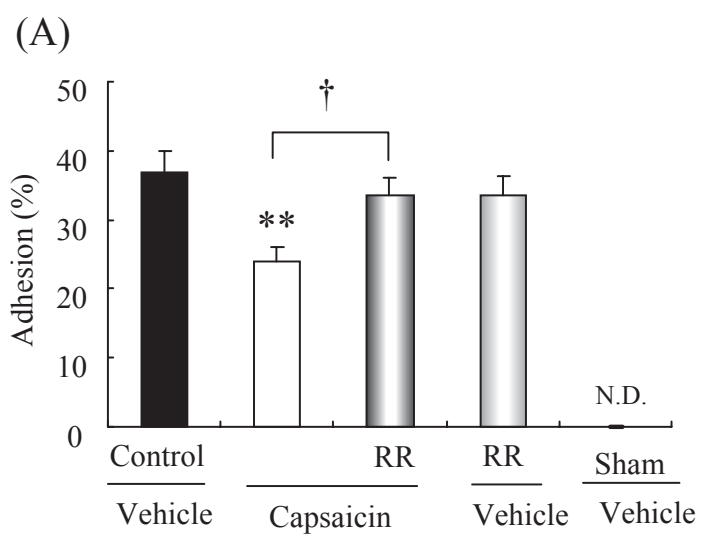

(B)

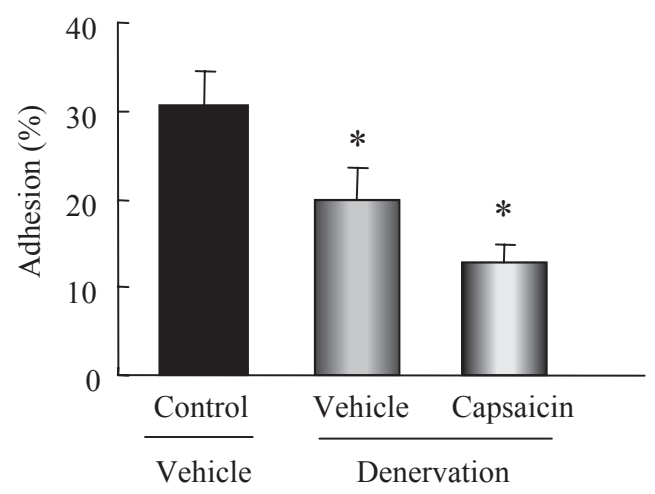

Fig. 4. Effect of TRP-channel antagonist and sensory denervation on talc-induced intestinal adhesion rats. A) Influence of ruthenium red $(\mathrm{RR}, 1 \mathrm{mg} / \mathrm{kg})$ on the preventive effect of capsaicin on talc-induced intestinal adhesion. Data are expressed as the mean \pm S.E.M. of 18 rats. ${ }^{*}$ Significantly different from the control group at $P<0.01$. 'Significantly different from the capsaicin-treated group at $P<0.05$ (PLSD test). B) Effect of sensory denervation on the preventive effect of capsaicin on talc-induced intestinal adhesion. Data are expressed as the mean \pm S.E.M. of 11 rats. *Significantly different from the control group at $P<0.05$ (PLSD test). 
(A) Control

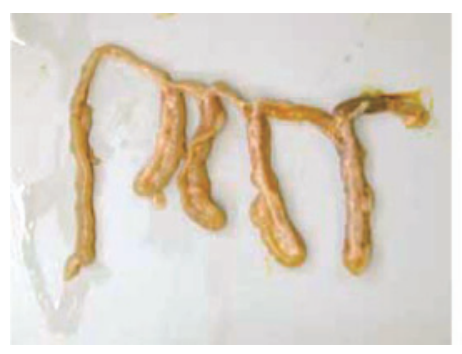

(C)

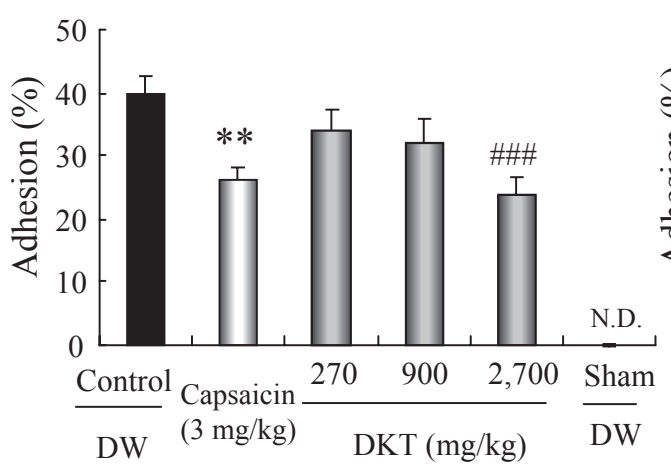

(B) DKT

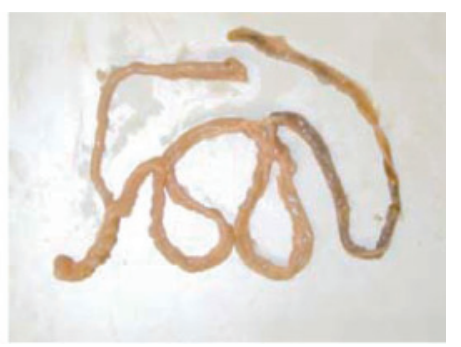

(D)

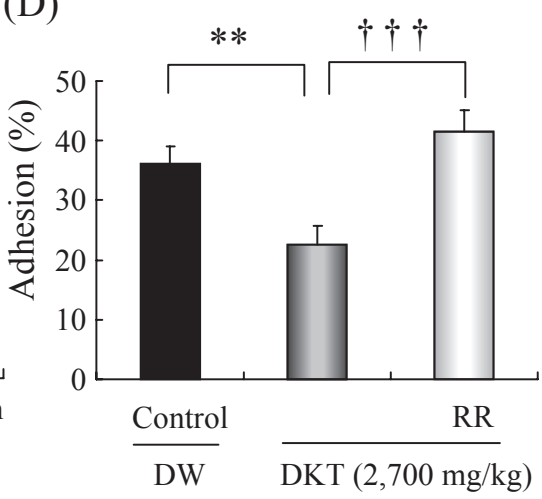

Fig. 5. Gross appearance of talc-induced intestinal adhesion in rats. Control (A) and DKT $(2,700 \mathrm{mg} / \mathrm{kg})$-treated rats (B) at 7 days after surgery. C) Effects of DKT on the adhesion rate on the talc-induced intestinal adhesion in rats. Data are expressed as the mean \pm S.E.M. of 16 rats. $* *$ Significantly different from the control group at $P<0.01$ (Student's $t$-test). ${ }^{\# \#}$ Significantly different from the control group at $P<0.001$ (Dunnett's test). D) Influence of RR on the preventive effect of DKT on the talc-induced intestinal adhesion. Data are expressed as the mean \pm S.E.M. of 12 rats. **Significantly different from the control group at $P<0.01$. "†ignificantly different from the DKT-treated group at $P<0.001$ (PLSD test). N.D., not detected.

\section{(A)}

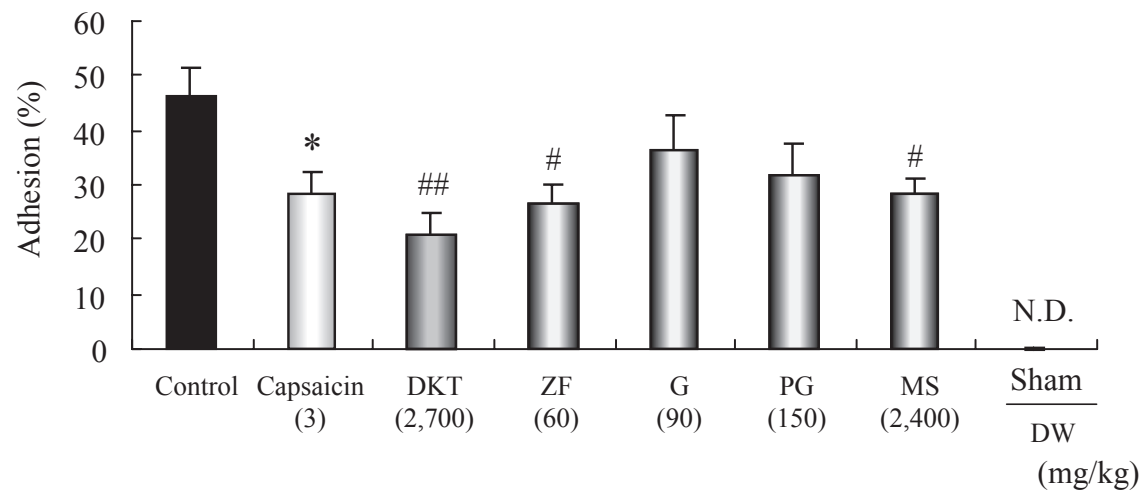

(B)

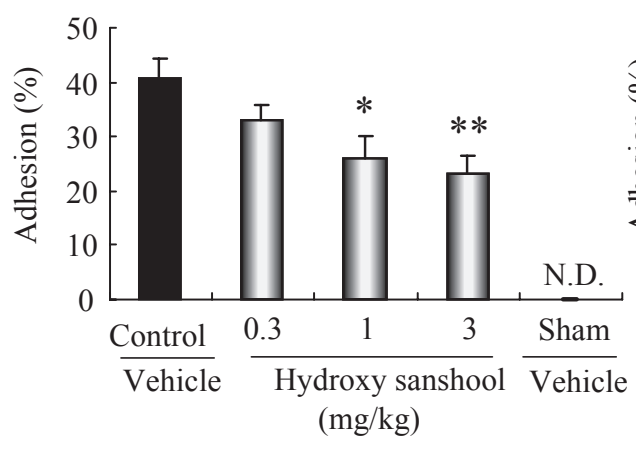

(C)

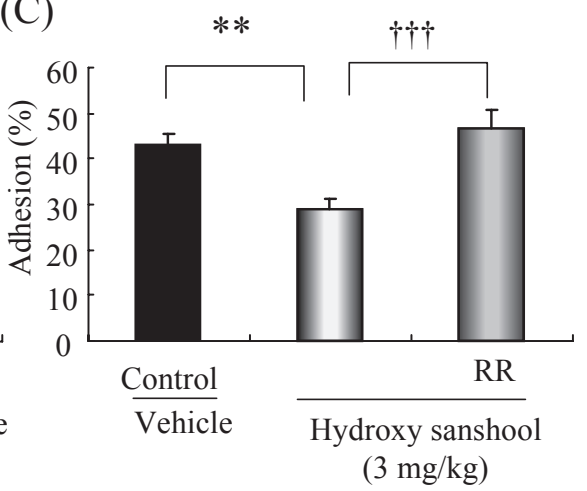

Fig. 6. Effects of constituent medical herbs of DKT and hydroxy sanshool on talc-induced intestinal adhesion rats. A) Effects of constituent medical herbs of DKT, zanthoxylum fruit (ZF), ginseng $(\mathrm{G})$, processed ginger $(\mathrm{PG})$, and maltose syrup (MS) on the adhesion rate on the talc-induced intestinal adhesion in rats. Data are expressed as the mean \pm S.E.M. of 10 rats. *Significantly different from the control group at $P<0.05$ (Student's $t$ test). ${ }^{\#} P<0.05$ and ${ }^{\#} P<0.01$, significantly different from the control group by Dunnett's test. B) Effects of hydroxy sanshool on the adhesion rate in the talcinduced intestinal adhesion in rats. Data are expressed as the mean \pm S.E.M. of 16 rats. $* P<0.05$ and $* * P<0.01$, significantly different from the control group by Dunnett's test. C) Influence of RR on the preventive effect of hydroxy sanshool (3 $\mathrm{mg} / \mathrm{kg}$ ) on the talc-induced intestinal adhesion. Data are expressed as the mean \pm S.E.M. of 12 rats. ${ }^{*}$ Significantly different from the control group at $P<0.01$. ${ }^{\dagger}$ Significantly different from the hydroxy sanshool-treated group at $P<0.001$ (PLSD test). N.D., not detected. 
istration of hydroxy sanshool at a dose of 1 and $3 \mathrm{mg} / \mathrm{kg}$ prevented the adhesion formation (the value being $23.2 \pm 3.0 \%$ and $25.9 \pm 4.2 \%$, respectively), but the effect of hydroxy sanshool (3 $\mathrm{mg} / \mathrm{kg})$ was completely inhibited by subcutaneous injection of RR (Fig. 6C).

\section{Discussion}

Postoperative adhesion formation begins with surgical trauma to the organ; the adhesion regions represent growth of sensory nerve fibers, and a proportion of these sensory nerves may be involved in various complications (7). In the present study, we identified localization of TRPV1 mRNA in the myenteric plexus and adhesion regions and found that TRPV1 channels may contribute to pathogenesis of the postoperative adhesion formation. Moreover, DKT and its component, hydroxy sanshool, prevented adhesion formation, and this action was abrogated by RR, an antagonist for certain ion channels including TRPV1.

It has been suggested that injury to tissues increases formation of fibrin matrices and infiltration of inflammatory cells, leading to formation of adhesion (1). The histological examination also confirmed the accumulation of fibrin and granulomatous inflammation in the adhesion regions after surgery. This inflammatory response is a cause of adhesion formation, although it is an indispensable reaction in wound healing.

In the gastrointestinal tract, the fibers expressing TRPV1 protein are distributed in a broad range of enteric nerves (mucosal, submucosal, and muscle layers) and are thought to be mainly of spinal cord origin $(24,25)$. Though the localization of TRPV1 mRNA has not been fully clarified, the mRNA has also been detected in longitudinal muscle-myenteric plexus preparations of stomachs (26). Furthermore, high TRPV1 expression has been detected in several inflammatory diseases of the colon and ileum, and neuropeptides released upon sensory nerve stimulation triggered by TRPV1 activation seem to play a role in intestinal disorders (9). Accordingly, in this study, we observed TRPV1 mRNA in the submucosal plexuses within muscle layers in sham-operated and talc-induced intestinal adhesion rats and in the myenteric plexus in talc-induced intestinal adhesion rats. It suggests that TRPV1 is up-regulated in the nerves under inflammatory conditions in talc-induced intestinal adhesion. Moreover, as demonstrated in Fig. 2D, we have detected an evident expression of TRPV1 mRNA in the adhesive regions formed between the separated loci of the intestinal tract. Adhesive regions usually contain a vast amount of extracellular matrices such as collagens and fibronectin as well as fibroblasts and recruited inflammatory cells. Because recent studies have shown that non-neuronal cells, such as mast cell and macrophages, have been also demonstrated to have an ability to express TRPV1 (27), we consider that the TRPV1 mRNA-expressing cells observed in the adhesion regions, at least some of them, may have non-neuronal origins. However, further studies are needed to clarify the character of the cell in adhesion regions. These findings address the possibility of TRPV1 involvement in the pathogenesis of postoperative adhesion. Then, to clarify the relationship between TRPV1 and formation of intestinal adhesions, the effect of a TRPV1 ligand, capsaicin, was examined.

Capsaicin, the pungent principle in hot pepper, which has been used as a neuropharmacological tool, excites predominantly TRPV1 located on sensory nerves. Stimulation of TRPV1 by capsaicin releases neuropeptides including calcitonin gene-related peptide, which modulate homeostasis in peripheral tissues via the axon reflex (9). To investigate the influence on adhesion formation, we evaluated the effect of capsaicin on talc-induced intestinal adhesion rats. We observed that orally administered capsaicin had a preventive effect on adhesion formation, and at doses of 1 and $3 \mathrm{mg} / \mathrm{kg}$, it significantly prevented adhesion formation compared to the control group, although the effects were not dose-dependent. To date, stimulation of sensory nerves by capsaicin application has been shown to participate in the maintenance of gastric mucosal homeostasis by regulation of neuropeptide release, gut motility, vascular function, and mucus/ $\mathrm{HCO}_{3}{ }^{-}$secretion; and this protection of the gastric mucosa by capsaicin has been called the 'neural emergency system' (11). In those reports, protection of mucosa was shown to be closely related to TRPV1-mediated up-regulation of the gastric blood flow. On the other hand, other researchers have demonstrated that mice lacking TRPV1 or several animal models with sensory denervation have attenuated gastrointestinal inflammation, such as dextran sulfate sodium-induced colitis and acid-induced gastroesophageal disease $(28,29)$. From these reports, the pharmacological effect of capsaicin was presumed to be based on the physiological functions of neuropeptides released from their peripheral endings or the TRPV1dependent desensitization of sensory nerves. To clarify whether the activation or desensitization of TRPV1 is involved in the prevention of intestinal adhesion, we investigated 1) the effect of injection of RR, a TRPV1 antagonist (30), on the adhesion-preventive action of capsaicin and 2) the effect of a large dose of capsaicin on the ablation of sensory nerves in postoperative intestinal adhesion. The effect of orally administered capsaicin was significantly inhibited by subcutaneous injection of RR, suggesting that the adhesion-preventive action of oral capsaicin is mediated by stimulation of sensory neurons 
via TRPV1. Furthermore, the desensitization of sensory nerves was not thought to occur in the present experimental setting because the rats treated with oral capsaicin for seven days showed wiping movement after application of $0.1 \%$ capsaicin to the eye. In the meanwhile, sensory denervation itself showed an adhesion-preventive effect. Further extensive investigations are necessary to reconcile the discrepancy observed in the present study; however, we will be able to discuss the following two points: 1) It seems possible that stimulation of TRPV1 is a two-edged sword in adhesion formation. TRPV1mediated enhancement of gut motility and blood flow might promote recovery from injury. In contrast, TRPV1 stimulation might result in neurogenic inflammation that exacerbates tissue damage. Differences in the timing, intensity, and biological context of TRPV1 simulation may produce diverse effects on intestinal adhesion. 2) The possible involvement of a pathophysiological/pharmacological target other than TRPV1 cannot be excluded. The RR, a frequently used capsaicin antagonist, is known to inhibit non-selectively various channels including TRPA1 (31). Capsaicin has also been known to have several bioactivities other than the agonistic activity to TRPV1 (32). Furthermore, the sensory denervation by capsaicin is used widely as a pharmacological tool, but it gives severe nerve damage (33), which is assumed to disrupt the physiologic activity. Therefore, to clarify whether TRPV1 plays some roles in adhesion formation, further extensive studies are necessary.

In Japan, DKT has been used to accelerate the recovery from postoperative ileus after abdominal surgery, and its clinical efficacy in post-surgical management has been demonstrated (15). We have already confirmed that DKT ameliorates gastrointestinal hypoperistalsis in the rat postoperative ileus model via cholinergic nerves and $5-\mathrm{HT}_{4}$ receptors and that zanthoxylum fruit and maltose syrup contribute to the ameliorating effect of DKT (34). We have already reported that hydroxy $\beta$-sanshool (35) and sanshools (36) contained in the zanthoxylum fruit of DKT constituents induce contraction of the guinea-pig ileum. The effect of hydroxy $\beta$-sanshools on gut smooth muscle contractility of hydroxy $\beta$-sanshool was abolished by a TRPV1 antagonist, capsazepine (35). In the present study, we demonstrated that orally administered DKT $(270-2,700 \mathrm{mg} / \mathrm{kg})$ dose-dependently prevented the adhesion formation in talc-induced intestinal adhesive obstruction rats; DKT at a dose of $2,700 \mathrm{mg} / \mathrm{kg}$ reduced the adhesion formation by approximately $40 \%$. To identify the active compound of DKT responsible for the adhesion-preventive action, the effects of the DKT constituents were examined. Zanthoxylum fruit and maltose syrup were found to contribute to this action, but processed ginger and ginseng did not markedly prevent ad- hesion formation. Thus, in this study we examined hydroxy sanshool, which is one of the most abundant unsaturated aliphatic acid amides in the methanol fraction of zanthoxylum fruit. Hydroxy sanshool showed a preventive effect on adhesion formation, and this effect was significantly inhibited by subcutaneous injection of RR. It has been reported that hydroxy sanshool has an agonistic activity on both TRPA1 and TRPV1 channels (21). On the other hand, a recent paper has suggested that hydroxy sanshool may increase the excitability of sensory neurons via inhibition of two-pore potassium channels (KCNK3, KCNK9, and KCNK18) (20). RR, an inorganic cation dye frequently used as a functional capsaicin antagonist $(30,31)$, inhibited the adhesion-preventive effect of DKT and hydroxyl sanshool, suggesting that TRPV1 may be involved in the effects. RR has been supposed to interact with TRPV1, TRPA1, and KCNK9 (31); however, the channels by which DKT and hydroxy sanshool exert their effects remain to be clarified in the future.

We previously reported that maltose syrup $(2,400$ $\mathrm{mg} / 10 \mathrm{~mL}$ per $\mathrm{kg}$ ), another active component of DKT, ameliorated delayed gastrointestinal transit in rat postoperative ileus and that the high osmotic pressure of the maltose syrup at that concentration was involved in the ameliorating effect (34). Thus, the improvement of gut function in the early postoperative period might contribute to the adhesion prevention. However, the role of maltose in this model is not well understood; therefore, clarifying this effect in postoperative adhesive obstruction is necessary.

In conclusion, this study demonstrated that the amount and localization of TRPV1 mRNA changed in talcinduced adhesion rats, and stimulation of TRPV1 channels attenuated the adhesion formation. Furthermore, denervation of sensory nerves by administration of a large dose of capsaicin has been found to decrease adhesion formation. These data suggest that TRPV1 and/or sensory nerves may play a complex, but critical, role in postoperative adhesion. Modulation of TRPV1 channels may offer a novel therapeutic strategy for prevention of postoperative adhesion obstruction. Elucidation of the target and mechanism of action of DKT will contribute to the development of such therapeutic options.

\section{References}

1 Liakakos T, Thomakos N, Fine PM, Dervenis C, Young RL. Peritoneal adhesions: etiology, pathophysiology, and clinical significance. Recent advances in prevention and management. Dig Surg. 2001;18:260-273.

2 Menzies D. Postoperative adhesions: their treatment and relevance in clinical practice. Ann R Coll Surg Engl. 1993;75:147153.

3 Vrijland WW, Jeekel J, van Geldorp HJ, Swank DJ, Bonjer HJ. 
Abdominal adhesions: intestinal obstruction, pain, and infertility. Surg Endosc. 2003;17:1017-1022.

4 Keltz MD, Peck L, Liu S, Kim AH, Arici A, Olive DL. Large bowel-to-pelvic sidewall adhesions associated with chronic pelvic pain. J Am Assoc Gynecol Laparosc. 1995;3:55-59.

5 Diamond MP, Freeman ML. Clinical implications of postsurgical adhesions. Hum Reprod Update. 2001;7:567-576.

6 Tokita Y, Yuzurihara M, Satoh K, Iizuka S, Imamura S, Kase Y, et al. Cholinergic nervous system plays an important role in rat postoperative intestinal adhesion. Surgery. 2008;143:226-232.

7 Sulaiman H, Gabella G, Davis C, Mutsaers SE, Boulos P, Laurent GJ, et al. Presence and distribution of sensory nerve fibers in human peritoneal adhesions. Ann Surg. 2001;234:256-261.

8 Richards AM, Mitsou J, Floyd DC, Terenghi G, McGrouther DA. Neural innervation and healing. Lancet. 1997;350:339-340.

9 Holzer P. Sensory neurone responses to mucosal noxae in the upper gut: relevance to mucosal integrity and gastrointestinal pain. Neurogastroenterol Motil. 2002;14:459-475.

10 Geppetti P, Trevisani M. Activation and sensitisation of the vanilloid receptor: role in gastrointestinal inflammation and function. Br J Pharmacol. 2004;141:1313-1320.

11 Holzer P. Neural emergency system in the stomach. Gastroenterology. 1998;114:823-839.

12 Abdel-Salam OM, Debreceni A, Mozsik G, Szolcsanyi J. Capsaicin-sensitive afferent sensory nerves in modulating gastric mucosal defense against noxious agents. J Physiol Paris. 1999;93: 443-454.

13 Immke DC, Gavva NR. The TRPV1 receptor and nociception. Semin Cell Dev Biol. 2006;17:582-591.

14 Yiangou Y, Facer P, Dyer NH, Chan CL, Knowles C, Williams $\mathrm{NS}$, et al. Vanilloid receptor 1 immunoreactivity in inflamed human bowel. Lancet. 2001;357:1338-1339.

15 Kono T, Kanematsu T, Kitajima M. Exodus of Kampo, traditional Japanese medicine, from the complementary and alternative medicines: is it time yet? Surgery. 2009;146:837-840.

16 Manabe N, Camilleri M, Rao A, Wong BS, Burton DD, Busciglio IA, et al. Effect of daikenchuto (TU-100) on gastrointestinal and colonic transit in humans. Am J Physiol Gastrointest Liver Physiol. 2010;298:970-975.

17 Hayakawa T, Kase Y, Saito K, Hashimoto K, Ishige A, Komatsu $\mathrm{Y}$, et al. Effects of Dai-kenchu-to on intestinal obstruction following laparotomy. J Smooth Muscle Res. 1999;35:47-54.

18 Tokita Y, Satoh K, Sakaguchi M, Endoh Y, Mori I, Yuzurihara M, et al. The preventive effect of Daikenchuto on postoperative adhesion-induced intestinal obstruction in rats. Inflammopharmacology. 2007; 15:65-66.

19 Kono T, Kaneko A, Hira Y, Suzuki T, Chisato N, Ohtake N, et al. Anti-colitis and -adhesion effects of daikenchuto via endogenous adrenomedullin enhancement in Crohn's disease mouse model. J Crohns Colitis. 2010;4:161-170.

20 Bautista DM, Sigal YM, Milstein AD, Garrison JL, Zorn JA, Tsuruda PR, et al. Pungent agents from Szechuan peppers excite sensory neurons by inhibiting two-pore potassium channels. Nat Neurosci. 2008;11:772-779.
21 Koo JY, Jang Y, Cho H, Lee CH, Jang KH, Chang YH, et al. Hydroxy-alpha-sanshool activates TRPV1 and TRPA1 in sensory neurons. Eur J Neurosci. 2007;26:1139-1147.

22 Michael GJ, Priestley JV. Differential expression of the mRNA for the vanilloid receptor subtype 1 in cells of the adult rat dorsal root and nodose ganglia and its downregulation by axotomy. J Neurosci. 1999;19:1844-1854.

23 Yonei Y, Holzer P, Guth PH. Laparotomy-induced gastric protection against ethanol injury is mediated by capsaicin-sensitive sensory neurons. Gastroenterology. 1990;99:3-9.

24 Nagy I, Santha P, Jancso G, Urban L. The role of the vanilloid (capsaicin) receptor (TRPV1) in physiology and pathology. Eur J Pharmacol. 2004;500:351-369.

25 Ward SM, Bayguinov J, Won KJ, Grundy D, Berthoud HR. Distribution of the vanilloid receptor (VR1) in the gastrointestinal tract. J Comp Neurol. 2003;465:121-135.

26 Schicho R, Florian W, Liebmann I, Holzer P, Lippe IT. Increased expression of TRPV1 receptor in dorsal root ganglia by acid insult of the rat gastric mucosa. Eur J Neurosci. 2004;19: 1811-1818.

27 Alawi K, Keeble J. The paradoxical role of the transient receptor potential vanilloid 1 receptor in inflammation. Pharmaco Ther. 2010;125:181-195.

28 Kihara N, de la Fuente SG, Fujino K, Takahashi T, Pappas TN, Mantyh CR. Vanilloid receptor-1 containing primary sensory neurones mediate dextran sulphate sodium induced colitis in rats. Gut. 2003;52:713-719.

29 Fujino K, de la Fuente SG, Takami Y, Takahashi T, Mantyh CR. Attenuation of acid induced oesophagitis in VR-1 deficient mice. Gut. 2006;55:34-40.

30 Amann R, Maggi CA. Ruthenium red as a capsaicin antagonist. Life Sci. 1991;49:849-856.

31 Czirják G, Enyedi P. Ruthenium red inhibits TASK-3 potassium channel by interconnecting glutamate 70 of the two subunits. Mol Pharmacol. 2003;63:646-652.

32 Luo XJ, Peng J, Li YJ. Recent advances in the study on capsaicinoids and capsinoids. Eur J Pharmacol. 2011;650:1-7.

33 Czaja K, Burns GA, Ritter RC. Capsaicin-induced neuronal death and proliferation of the primary sensory neurons located in the nodose ganglia of adult rats. Neuroscience. 2008;154:621630.

34 Tokita Y, Yuzurihara M, Sakaguchi M, Satoh K, Kase Y. The pharmacological effects of Daikenchuto, a traditional herbal medicine, on delayed gastrointestinal transit in rat postoperative ileus. J Pharmacol Sci. 2007;104:303-310.

35 Satoh K, Hashimoto K, Hayakawa T, Ishige A, Kaneko M, Ogihara S, et al. Mechanism of atropine-resistant contraction induced by Dai-kenchu-to in guinea pig ileum. Jpn J Pharmacol. 2001;86:32-37.

36 Hashimoto K, Satoh K, Kase Y, Ishige A, Kubo M, Sasaki H, et al. Modulatory effect of aliphatic acid amides from Zanthoxylum piperitum on isolated gastrointestinal tract. Planta Medica. 2001;67:179-181. 